\title{
Demonstration of an Exponential Advantage in Communication Complexity via the Quantum Switch
}

\author{
Nora Tischler, ${ }^{1, *}$ Kejin Wei, ${ }^{2,3}$ Si-Ran Zhao, ${ }^{2,3}$ Yu-Huai Li, ${ }^{2,3}$ Juan Miguel Arrazola, ${ }^{4,5}$, \\ Yang Liu, ${ }^{2,3}$ Weijun Zhang, ${ }^{6} \mathrm{Hao} \mathrm{Li},{ }^{6}$ Lixing You, ${ }^{6}$ Zhen Wang, ${ }^{6}$ Yu-Ao Chen,,${ }^{2,3}$ \\ Barry C. Sanders, ${ }^{2,7,8}$ Qiang Zhang, ${ }^{2,3}$ Geoff J. Pryde, ${ }^{1}$ Feihu Xu ${ }^{2,3}$ and Jian-Wei Pan ${ }^{2,3}$ \\ ${ }^{1}$ Centre for Quantum Dynamics, Griffith University, Brisbane QLD 4111, Australia \\ ${ }^{2}$ Shanghai Branch, Hefei National Laboratory for Physical Sciences at Microscale and Department of Modern Physics, \\ University of Science and Technology of China, Shanghai 201315, China \\ ${ }^{3}$ CAS Center for Excellence and Synergetic Innovation Center in Quantum Information and Quantum Physics, \\ University of Science and Technology of China, Shanghai 201315, China \\ ${ }^{4}$ Xanadu, 372 Richmond Street W, Toronto, Ontario M5V 1X6, Canada \\ ${ }^{5}$ Centre for Quantum Technologies, National University of Singapore, 3 Science Drive 2, Singapore 117543 \\ ${ }^{6}$ State Key Laboratory of Functional Materials for Informatics, Shanghai Institute of Microsystem and Information Technology, \\ Chinese Academy of Sciences, Shanghai 200050, China \\ ${ }^{7}$ Institute for Quantum Science and Technology, University of Calgary, Alberta T2N 1N4, Canada \\ ${ }^{8}$ Program in Quantum Information Science, Canadian Institute for Advanced Research, Toronto, Ontario M5G 1M1, Canada \\ n.tischler@griffith.edu.au
}

\begin{abstract}
The quantum switch is a tool that allows coherently controlling the order in which two operations are applied. We report the first experimental demonstration of the exponential advantage in communication complexity enabled by this tool. (C) 2019 The Authors
\end{abstract}

\section{Introduction}

Coherent superposition fundamentally underpins quantum effects. For example, the superposition of quantum states famously gives rise to the nonclassical phenomena of entanglement and nonorthogonality. Recently, the notion of a coherent superposition of the order of quantum gates has been explored [1]. Such a superposition can be realized with the so-called quantum switch, where a control qubit determines in what order two transformations are performed on a target system [2]. The process is causally ordered when the control qubit is in either the logical $|0\rangle$ or $|1\rangle$ state, whereas it is causally indefinite when the control qubit is in a superposition. Indefinite causal order has diverse applications, from developing quantum theories of gravity [1], to the reduction of query complexity in quantum computation [3,4]. It has also been theoretically established that the quantum switch can provide an advantage in the context of communication complexity $[5,6]$.

The field of communication complexity studies the minimum amount of information that must be transmitted in order to compute functions that depend on inputs which are distributed between remote parties [7]. It has applications in distributed computing, streaming algorithms, very-large-scale integration, and more. Quantum resources, such as entanglement and nonorthogonality, can offer an advantage in communication complexity over classical resources [7]. In the best case, quantum resources can allow an exponential reduction in the scaling of the required communication as a function of the problem size.

In our work [8], we realize such an exponential advantage in a communication complexity task by using a quantum switch. Our photonic experiment requires less than $(0.696 \pm 0.006)$ times the communication of any causally ordered protocol. Our experiment also constitutes the first implementation of a quantum switch with a multi-qubit target system.

The task we perform was introduced in Ref. [6] under the name exchange evaluation game. In this task, there are three remote parties, Alice, Bob, and Charlie. Alice and Bob receive inputs $(\boldsymbol{x}, f)$ and $(\boldsymbol{y}, g)$, respectively, where $\boldsymbol{x}, \boldsymbol{y} \in\{0,1\}^{n}$ are $n$-bit strings and $\mathrm{f}, \mathrm{g}:\{0,1\}^{n} \rightarrow\{0,1\}$ are Boolean functions. The third party, Charlie, needs to compute the exchange evaluation function $E E(\boldsymbol{x}, f, \boldsymbol{y}, g)=f(\boldsymbol{y}) \oplus g(\boldsymbol{x})$. In Ref. [6], Guérin and coworkers proposed a protocol (illustrated in Fig. 1(a)) that uses the superposition of the communication direction between Alice and Bob to solve the exchange evaluation game with unit success probability, using an amount of communication that scales exponentially better with the input bit string size $n$ compared to any causally ordered protocol. We experimentally implement a variant of this protocol, which includes modifications that render it experimentally feasible with current technology.

\section{Experiment}

\subsection{Protocol}

In our protocol, we use a quantum switch where one qubit acts as the control, and the target system is $2^{n+1}$ dimensional, which makes it equivalent to $(n+1)$ qubits. We implement both systems in a heralded single photon, generated through spontaneous parametric down-conversion, by encoding the control system in the 
path degree of freedom and the target system in the arrival time of the photon, which is divided into a set of discrete time bins. For the protocol, the control qubit is initialized in a superposition state, i.e. a superposition of two paths, while the target system is initialized as the photon being in the first time bin. Then two unitary transformations, $U_{\mathrm{A}}$ and $U_{\mathrm{B}}$, are applied on the target system in an order that depends on the path. Each of these unitaries consists of a phase modulation followed by a time delay, where the specifics depend on Alice's inputs $(\boldsymbol{x}, f)$ for $U_{\mathrm{A}}$ and Bob's inputs $(\boldsymbol{y}, g)$ for $U_{\mathrm{B}}$. The experimental implementation uses a fiberbased Sagnac interferometer, where the photon interferes such that it exits at one or the other of the two interferometer outputs, depending on the binary answer to the exchange evaluation game. More details are provided in Ref. [8].

\subsection{Experimental Results}

The causally indefinite protocol is tested with several values of the system size $n$. Fig.1(b) shows the experimental transmitted information for different system sizes. We compare our protocol (in red) with a bound for classical protocols (gray solid curve) and a bound for quantum causally definite protocols (blue solid curve). Fig.1(b) indicates that, with the increase of system size $n$, the causally indefinite protocol provides an exponential advantage in the scaling of transmitted information. In particular, for $n=12$, the experimental results clearly beat all classical protocols as well as all quantum causally definite protocols.

(a)

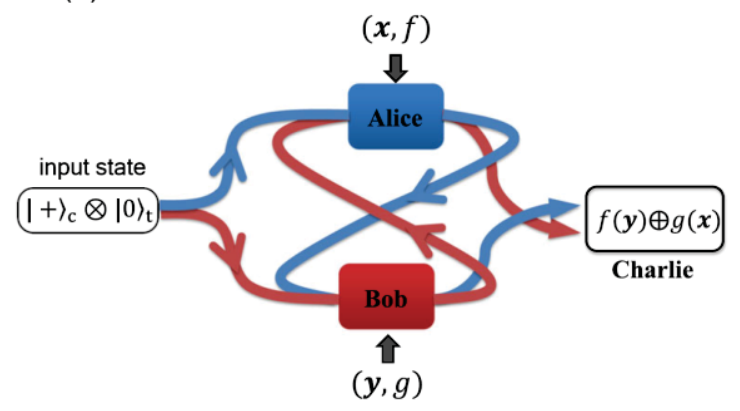

(b)

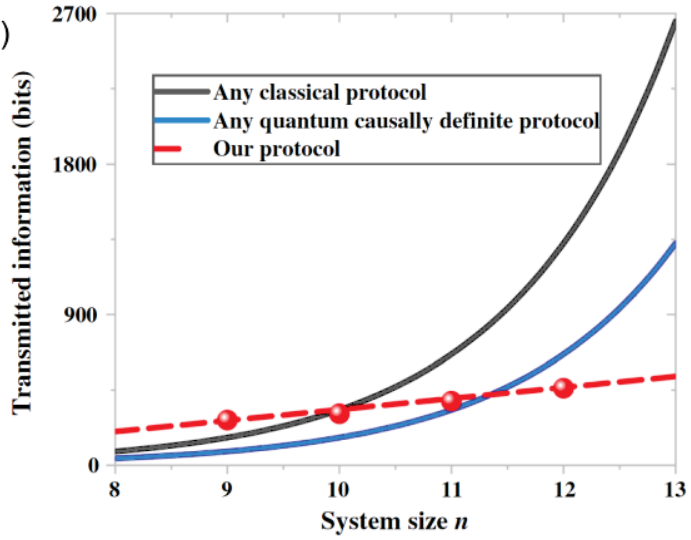

Fig. 1. (a) Concept behind the quantum protocol for solving the exchange evaluation game. (b) Experimental results. Transmitted information as a function of the system size for our protocol, and bounds for causally definite protocols. Images adapted from Ref. [8].

\section{Conclusion}

Unlike past experimental implementations of the quantum switch, our experiment involves a multi-qubit target system. We achieve this through the use of a high-dimensional degree of freedom, the photon arrival time. Encoding the target qubits in the photon arrival time leads to an exponential number of time bins as a function of the number of target qubits. Nevertheless, an exquisite control over time bins has allowed us to realize a target system which is equivalent to 13 qubits and lets us test $n=12$. In this way, we show that our causally indefinite quantum protocol can outperform any causally definite protocol, classical or quantum. Thus, our experiment demonstrates that the superposition of communication direction can serve as a powerful resource in communication complexity.

\section{References}

[1] Č. Brukner, “Quantum causality”, Nat. Phys. 10, 259-263 (2014).

[2] G. Chiribella, G. M. D'Ariano, P. Perinotti, and B. Valiron, "Quantum computations without definite causal structure", Phys. Rev. A 88, 022318 (2013).

[3] M. Araújo, F. Costa, and Č. Brukner, "Computational advantage from quantum-controlled ordering of gates", Phys. Rev. Lett. 113, 250402 (2014).

[4] L. M. Procopio et al., "Experimental superposition of orders of quantum gates", Nat. Commun. 6, 7913 (2015).

[5] A. Feix, M. Araújo, and Č. Brukner, "Quantum superposition of the order of parties as a communication resource", Phys. Rev. A 92, 052326 (2015).

[6] P. A. Guérin, A. Feix, M. Araújo, and Č. Brukner, "Exponential communication complexity advantage from quantum superposition of the direction of communication", Phys. Rev. Lett. 117, 100502 (2016).

[7] H. Buhrman, R. Cleve, S. Massar, and R. de Wolf, "Nonlocality and communication complexity", Rev. Mod. Phys. 82, 665 (2010).

[8] K. Wei et al., "Experimental quantum switching for exponentially superior quantum communication complexity", Phys. Rev. Lett. 122, 120504 (2019). 